\title{
From BPEL Processes to YAWL Workflows`
}

\author{
Antonio Brogi and Razvan Popescu \\ Computer Science Department, University of Pisa, Italy
}

\begin{abstract}
BPEL is currently the most widespread language for composing Web services, but it lacks formal semantics. YAWL is a workflow language with a well defined formal semantics that implements the most common workflow patterns. In this paper we provide a methodology for translating BPEL processes into YAWL workflows, thus paving the way for the formal analysis, aggregation and adaptation of BPEL processes. The approach we propose defines a YAWL pattern for each BPEL activity. The translation of a BPEL process reduces then to suitably instantiating and interconnecting the patterns of its activities.
\end{abstract}

\section{Introduction}

The service-oriented computing paradigm [9] uses services as building blocks for developing future heterogeneous, distributed applications. Two main reasons for composing (Web) services are the need for rapid application development, and the need to answer complex queries that cannot be satisfied by one service alone.

WSDL 12 is the current standard for describing Web service interfaces, yet it provides only a syntactic description of the supported operations. This severely affects the process of (semi-)automated service composition as composed services may lock during their interaction.

BPEL 2] has emerged as a language for expressing Web service compositions. A BPEL process provides the behaviour of a Web service in terms of coordinating one or more WSDL services. A downside of BPEL is that clients of the business process are in charge of manually selecting the services to be composed, and of building the composite service. Furthermore, BPEL lacks a formal semantics and hence it does not provide suitable means for the analysis of service compositions.

YAWL [10] is a new proposal of a workflow/business processing system that supports a concise and powerful workflow language and handles complex data transformations and Web service integrations. As it implements the most common workflow patterns, YAWL can be used as a lingua franca for expressing the behaviour of Web services (described using BPEL or OWL-S [8], for example). Despite its graphical nature, YAWL has a well defined formal semantics. It is a state-based language and the semantics of a workflow specification is defined as a transition system. Furthermore, being based on Petri nets, it provides a firm basis for the formal analysis of real-world services.

Our long-term goal is to provide a methodology for the (semi-)automated aggregation and adaptation of Web services into new heterogeneous applications.

\footnotetext{
* This work has been partially supported by F.I.R.B. project TOCAI.IT.
} 
To cope with the previous issues we argue for the use of service contracts 4] consisting of (a) a (WSDL) signature, (b) an (OWL) ontological description, and (c) a (YAWL) behaviour (or protocol). The signature and the ontological information serve for enhancing the service discovery process and for overcoming signature mismatches. The protocol information can be employed for generating the behaviour of the aggregated service and for verifying properties of the aggregate (such as lock freedom), as well as for coping with behavioural mismatches.

In [4] we described a core aggregation process for composing YAWL services. The core aggregation process inputs a set of service contracts to be aggregated and it outputs the contract of the aggregated service. The control-flow of the aggregate is built, on the one hand, from the initial control-flow of the participant services, and on the other hand, from data-flow dependencies obtained by semantically matching service parameters. This paper complements 4 by devising a methodology for translating BPEL processes into YAWL workflows. As a result, BPEL services can be translated into YAWL workflows, then aggregated, and finally deployed as a new BPEL service. It is worth stressing the importance of the last two features. As we will see, handling synchronisation links, scope activities, events, faults, and compensations, sensibly complicates the translation. Probably because of their complexity, these mechanisms have not usually been considered by the formalisations of BPEL that have been proposed so far (e.g., 6[1]). On the other hand, since these features are indeed exploited in real BPEL descriptions, and do contribute to the expressiveness of "real" BPEL, we argue that they cannot be ignored.

The translation approach we describe here defines a YAWL pattern for each BPEL activity, as well as for a whole BPEL process. The role of an activity pattern is twofold - to provide a unique representation of the activity, and to provide an execution context for it. Given a BPEL process, the approach automatically generates its YAWL translation by:

1. Instantiating the pattern of each activity defined in the BPEL process, and

2. Suitably connecting the obtained patterns into the final workflow.

The main features of the translation methodology can be summarised as follows:

- It is a pattern-based, compositional approach,

- It copes with all types of BPEL activities, and

- It handles events, faults and (explicit) compensation.

\section{A Brief Introduction to BPEL and YAWL}

The next two Subsections give a very high-level view of both languages. Some other details on the two languages will be discussed in the next Section, while describing the translation methodology. For a complete description of the two languages, please see [2] for BPEL, and [10] for YAWL. 


\subsection{BPEL: Business Process Execution Language}

BPEL is a language for expressing the behaviour of a business process. It enables the specification of control and data logic around a set of Web service interactions. A BPEL process exposes a WSDL interface to its clients.

A BPEL process can be either abstract or executable. An abstract process hides implementation details (i.e., private information), while an executable process provides the full interaction behaviour.

BPEL defines the notion of partner link to model the interaction between a business process and its partners. A partner link refers to at most two WSDL port types, one of the interface to the business process (viz., operations offered by the process to the partner), and the other of the interface of a partner (viz., operations offered by the partner to the business process).

BPEL is a hybrid language that combines features from both the blockstructured language XLANG and from the graph-based language WSFL. The former contributed with basic activities (e.g., for sending and receiving messages, for waiting for a period of time, and so on) as well as with structured ones (e.g., sequential or parallel execution of activities, activity scoping, and so on) for combining activities into complex ones. The latter brought the definition of links to synchronise activities executed in parallel. Other features of BPEL are instance management through correlation sets, event and fault handling, as well as compensation capabilities.

The BPEL basic activities are: receive/reply through which a BPEL process inputs/sends a message from/to a partner service, invoke through which a BPEL process asynchronously/synchronously invokes an operation of a partner service, wait for delaying the execution of the process, throw for signalling faults, terminate for explicitly terminating the execution of the process, a dummy empty for doing a "no-op", assign for copying values between variables, and compensate for invoking a compensation handler.

The structured activities are: sequence, switch, and while for sequential, conditional and repeated activity execution, flow for parallel activity execution, pick for managing the non-deterministic choice of the activity to be executed, and scope for providing an execution context for an activity.

\subsection{YAWL: Yet Another Workflow Language}

YAWL is a new proposal of a workflow/business processing system, which supports a concise and powerful workflow language and handles complex data transformations and Web service integration. YAWL defines twenty most used workflow patterns divided in six groups - basic control-flow, advanced branching and synchronisation, structural, multiple instances, state-based, and cancellation. A thorough description of these patterns may be found in [11].

YAWL extends Petri Nets by introducing some workflow patterns (for multiple instances, complex synchronisations, and cancellation) that are not easy to express using (high-level) Petri Nets. Being built on Petri Nets, YAWL is an easy to understand and to use formalism, which features an intuitive (graphical) representation of services. Moreover, it can benefit from the abundance 
of Petri net analysis techniques. With respect to the other workflow languages (mostly proposed by industry), YAWL relies on a well-defined formal semantics based on transition systems. Moreover, not being a commercial language, YAWL supporting tools (editor, engine) are freely available.

From a control-flow perspective, a YAWL file describes a workflow specification that consists of a tree-like structure of extended workflow nets (or EWF-nets for short). An EWF-net is a graph where nodes are tasks or conditions, and edges define the control-flow relation. Each EWF-net has a single input condition and a single output condition.

Tasks employ one join and one split construct, which may be one of the following: AND, OR, XOR, or EMPTY. Intuitively, the join of a task $T$ specifies "how many" tasks before $T$ are to be terminated in order to execute $T$, while the split construct specifies "how many" tasks following $T$ are to be executed.

It is worth noting that YAWL tasks may be interpreted as Petri net transitions, and YAWL conditions can be represented as Petri net places. The controlflow for tasks with $\mathrm{XOR} / \mathrm{OR}$ splits is managed through predicates in the form of logical expressions. When a task finishes its execution, it places tokens in its output places, depending on its split type. Dually, a task is enabled for execution depending on its join and on the tokens available in its input places.

Another feature of YAWL is the use of cancellation sets consisting of conditions and tasks. When a task is executed all tokens from its cancellation set (if any) are removed.

From a data-flow perspective, YAWL uses XMLSchema, XPath and XQuery for dealing with data. Variables are defined at both EWF-net and task levels, and bindings between them are realised through XQuery expressions.

\section{From BPEL to YAWL}

The objective of this paper is to present a methodology for translating BPEL processes into YAWL workflows. First, we define a YAWL pattern for each BPEL activity, as well as for the entire business process. Then, the workflow corresponding to a BPEL process is obtained by suitably instantiating and interconnecting the workflows of all its activities.

Subsections 3.1 and 3.2 introduce the basic pattern template and the structured pattern template, which are used to define the patterns of the basic and structured activities, respectively. Subsection 3.3 defines the process pattern and describes the process of obtaining the final workflow.

In the following we shall use the term pattern template to refer to the pattern of a generic BPEL activity (viz., either basic or structured). The role of a pattern template is twofold: It provides the necessary elements for uniquely identifying an activity/process, as well as an execution context for the translated activity/process.

\subsection{The Basic Pattern Template}

BPEL uses structured activities to specify the order in which activities have to be executed. For example, the second activity in a sequence can be executed only 


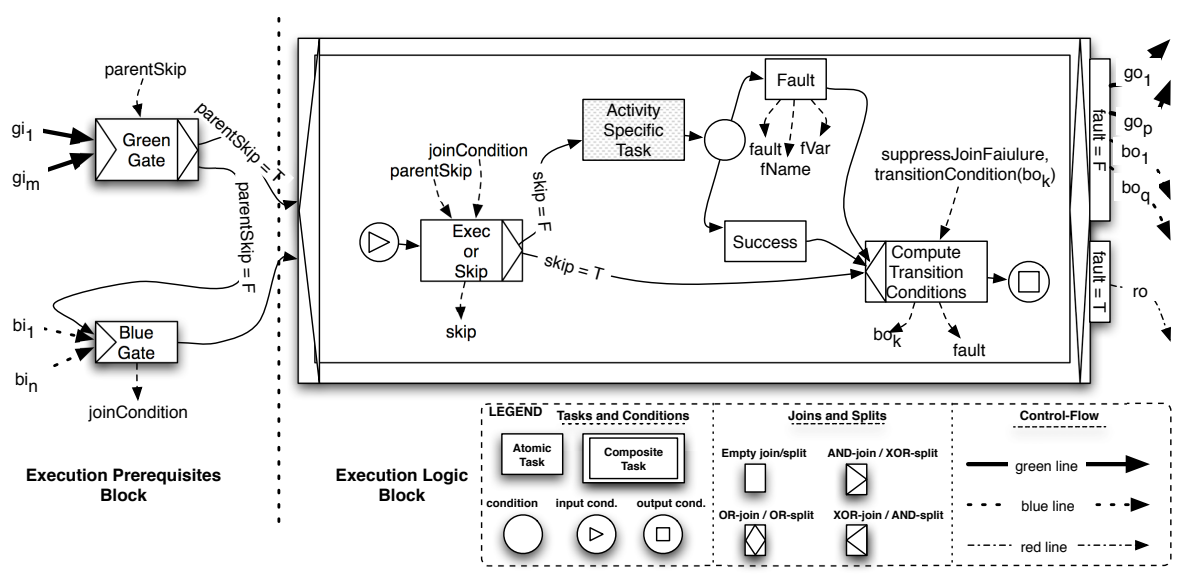

Fig. 1. The basic pattern template

when the first has finished its execution. Moreover, the flow construct allows for synchronisation links to be defined among activities. When an activity is structurally enabled, BPEL waits for the statuses of all its incoming links (if any) to be determined. At that point BPEL computes the joinCondition (a logical expression), which guards the execution of the activity. A true value leads to the execution of the activity, while a false value leads to either raising a joinFailure fault, or to skipping the entire activity. It is important to note that a structured activity that is skipped leads to skipping all the activities nested within it. Skipping an activity leads to propagating negative (viz., false) statuses on its output links. This process is called dead-path-elimination.

We model the structural relations among BPEL activities through what we call green lines. A pattern has one or more green inputs, which are used to enable it from the structural point of view. Dually, it has one or more green outputs, to be sent upon completion of the pattern, which will be used to enable other patterns. For example, the patterns translating child activities of a BPEL sequence have to be linked through green lines. On the other hand, we model the synchronisation links among BPEL activities using blue lines. A pattern has one blue input for each synchronisation link that targets the activity it translates. Analogously, it has one blue output for each link that emerges from the activity it translates. For example, inside a BPEL flow, a synchronisation link from activity $A$ to activity $B$ is translated into a blue line from the pattern translating $A$ to the pattern translating $B$. Finally, in order to cope with faults we use red lines. Patterns that treat errors have red inputs, while patterns that generate errors have red outputs. For example, the translation of the BPEL throw activity has to have a red line as output, while the translation of the BPEL fault handler should input a red line.

The basic pattern template is illustrated in Figure 1. It consists of an Execution Prerequisites Block and of an Execution Logic Block. Green input lines of a 
pattern are denoted by $g i$, and green outputs by go. Similarly, $b i$ and bo denote blue inputs and ouputs, and $r i$ and ro red ones.

The Execution Prerequisites Block (EPB). The EPB is in charge of enabling the pattern. In order to execute, a pattern has to be enabled both from the structural and from the synchronisation point of view.

The Green Gate task of the EPB is in charge of waiting for the green tokens. It also inputs a parentSkip boolean variable from its parent 11 activity, whose value indicates whether the latter is skipped or not. Indeed, since each structured activity could be skipped if it is the target of a synchronisation link, it outputs a parentSkip variable to all the patterns corresponding to its nested (child) activities.

If parentSkip holds true then the pattern must be skipped, as one of its ancestors was skipped. In this case GreenGate will immediately enable the Execution Logic Block, without having to wait for the statuses of its incoming links to be computed. If instead parentSkip holds false then the pattern is ready to be executed from the structural viewpoint. In this case, the execution of the EPB continues with the BlueGate task, which waits for all blue tokens and then it computes the value of the joinCondition by taking into account the statuses of its incoming links stored into bi boolean variables. Then, the BlueGate enables the Execution Logic Block.

The Execution Logic Block (ELB). The ELB has three possible behaviours: It can execute successfully, it can be silently skipped, or it can raise a fault. The third behaviour corresponds to a false joinCondition (see next) or to an erroneous execution of the activity.

The ExecOrSkip task of ELB computes the skipping condition (into the skip boolean variable) as a logical disjunction between the parentSkip and the negation of the joinCondition variables. Indeed, an activity is skipped either since one of its ancestors was skipped (parentSkip=true), or since its joinCondition is false. If skip evalutes to false then the ActivitySpecificTask is executed, otherwise the ComputeTransitionConditions task is executed.

The ActivitySpecificTask is the key task of the pattern. It uniquely identifies the translated activity and it provides the computations needed by the activity. Instantiating the basic pattern template for a particular activity consists of equipping the ActivitySpecificTask with a name identifying the activity, and with the inputs and outputs defined by the activity. For example, the Wait pattern has an ActivitySpecificTask called Wait that inputs the duration of the wait.

The execution of the ActivitySpecificTask is simlutated through the deferred choice consisting of the Fault and Success tasks, together with their input place. The environment (viz., the "client" of the workflow) will determine whether Fault or Success will be executed. The execution of the Fault task corresponds to an erroneous execution of the activity (e.g., a receive activity has received an incorrect message). The Fault task outputs the name and data associated with

${ }^{1}$ When an activity $A$ is directly nested within a structured activity $S$, we also say that $S$ is the parent of $A$ and that $A$ is a child of $S$. 
the fault, and it sets the boolean fault flag to true. Dually, Success corresponds to a successful execution of the activity. It is important to note that the deferred choice must be defined only for activities whose execution may be erroneous (e.g., receive, invoke, and so on). Otherwise, the ActivitySpecificTask is directly connected to the ComputeTransitionConditions task.

BPEL uses the suppressJoinFailure attribute to determine the process behaviour when the joinCondition is false. If the suppressJoinFailure attribute corresponding to an activity (defined by it or by one of its ancestors) is set to $N O$, the BPEL engine raises a joinFailure fault. Otherwise, it employs the deadpath-elimination by propagating negative statuses on all its output links. The ComputeTransitionConditions task concludes the execution of the ELB and of the pattern. On the one hand, it computes the status of each output (synchronisation) link, as defined by the transitionCondition attribute of the respective BPEL link. Link statuses are stored into bo variables, which have to be mapped onto $b i$ variables of other patterns when constructing the workflow of the business process. On the other hand, it signals a joinFailure by setting the fault flag to true in case of a false joinCondition if the corresponding suppressJoinFailure attribute is set to $N O$.

Upon completion, the ELB outputs green and blue tokens if and only if the pattern was successfully executed. Dually, it outputs a red token if and only if a fault was raised.

BPEL Basic Activities. Space limitations do not allow us to present the patterns of all the basic BPEL activities. We shall resume to presenting some general guidelines for customising and instantiating the basic pattern template.

In order to obtain the pattern of a basic activity, one has to (1) customise the ActivitySpecificTask, and (2) remove the deferred choice controlling the success of the activity if the activity cannot have an erroneous execution, as well as (3) set the (maximum) number of inputs and outputs of the pattern. The customisation of the ActivitySpecific Task regards the name of the task, which has to identify the pattern, as well as the inputs and the outputs of the task, which are obtained from the inputs and the outputs of the BPEL activity. Note that a pattern has at least one green input and one green output.

The Invoke, Receive and Reply patterns all have one green input. Invoke and Reply patterns have only one green output (for the pattern of the following activity, if any), while Receive can have at most two green outputs (the second to enable the pattern for event handling of the entire business process, if the createInstance attribute of the BPEL receive is set to yes).

The patterns Throw, Wait, Terminate and Empty have one green input and one green output, and they do not need the deferred choice block, as their execution cannot be erroneous. The ActivitySpecificTask will be hence directly connected to the ComputeTransitionConditions task. Note that a fault raised by a Throw pattern is not considered as an erroneous execution of the throw activity. Some other particularities are that the ActivitySpecificTask of the Wait pattern invokes the YAWL TimeService in order to delay the execution of the workflow, while the successful execution of a Terminate pattern leads to the cancellation 
of all tokens inside the pattern translating the process activity. (Further details on the latter will be given later on when describing the process pattern.)

The assign and the compensate activities are treated as structured patterns, as we will see in the next Subsection.

\subsection{The Structured Pattern Template}

A BPEL structured activity defines one or more activities to be executed in a certain order. In order to cope with this, we define the structured pattern template as a tuple consisting of a Begin pattern, an End pattern, as well as of a Pattern Template for each child activity.

The purpose of the Begin and End patterns is to provide an identification for the activity being translated. More importantly, the execution of Begin logically corresponds to the initiation of the structured activity (as a whole), whereas the execution of End logically marks the termination of the structured activity.

Both Begin and End patterns are generated from the basic pattern template, and they are quite similar to the Empty pattern. On the one hand, Begin is in charge of enabling the structured pattern both from the structural and synchronisation viewpoints. Hence, Begin has to input the green and the blue lines and to raise a joinFailure in case of a false joinCondition if the corresponding suppressJoinFailure attribute is set to NO. Furthermore, it provides a green output for each PatternTemplate corresponding to a child activity that can be executed first. On the other hand, End has to wait for the green tokens from all PatternTemplates of the child activities that have to be executed last. Moreover, End is the source of the blue outputs corresponding to synchronisation links having as source the structured activity. In general, End cannot lead to any fault being raised, and hence it does not have a red output.

A structured activity introduces a new nesting level and consequently Begin has to output a parentSkip variable to the patterns of all the (child) activities nested inside the structured one, as well as to End. In this way we achieve the dead-path-elimination inside structured patterns.

Now, the patterns of all structured activities are obtained by adjusting the Begin and End patterns and by suitably interconnecting them with the PatternTemplates. Basically, both processes depend on the way in which the structured activity enables for execution its child activities. In the following we shall write $\operatorname{Begin}(X)$ and $\operatorname{End}(X)$ to refer to the Begin and End patterns of a structured activity $X$.

BPEL Structured Activities. Space limitations do not allow us a detailed description of all the structured patterns. However, we shall try to describe the most relevant features of each pattern.

The Sequence, Switch, Flow and Pick patterns all share the same structure:

\begin{tabular}{|c|c|c|c|c|}
\hline Sequence & $\rightarrow$ & Begin(Sequence) & PatternTemplate $^{+}$ & End(Sequence $)$ \\
\hline Switch & $\rightarrow$ & Begin(Switch) & PatternTemplate $^{+}$ & $\operatorname{End}($ Switch $)$ \\
\hline Flow & $\rightarrow$ & Begin(Flow) & PatternTemplate ${ }^{+}$ & $\operatorname{End}($ Flow $)$ \\
\hline Pick & $\rightarrow$ & $\operatorname{Begin}($ Pick) & PatternTemplate $^{+}$ & End (Pick) \\
\hline
\end{tabular}


The Sequence pattern consists of a Begin and an End pattern, together with at least one Pattern Template. Begin(Sequence) must enable the execution of only the first Pattern Template in the sequence, each Pattern Template enables the next one in the sequence, and End[Sequence] must wait for the last Pattern Template to finish its execution.

The Switch pattern includes one Pattern Template for each conditional branch, and each Pattern Template must verify the guard condition of the corresponding branch. A false guard leads to skipping the corresponding branch and hence to dead-path-elimination inside the corresponding pattern. The Pattern Templates are linked in the order in which the conditional branches occur in the switch activity. If no otherwise branch is defined, a default one with an empty activity guarded by an (always) true condition is considered.

A flow activity concurrently executes a bag of activities among which synchronisation links can be defined. Begin(Flow) has to enable the patterns of all its child activities, and hence it has one green output for each PatternTemplate. Dually, the execution of End(Flow) is delayed until all PatternTemplates finish their execution.

A pick basically waits for a message or an alarm event to take place. Its pattern is slightly more complicated due to the fact that the first event that is triggered causes all other events to be cancelled. Begin(Pick) mainly differs from $B e-$ gin(Flow) in that its ActivitySpecificTask is a composite task in charge of branch selection. Moreover, each Pattern Template of the pick has a guard condition that checks whether its branch id matches the id of the branch selected in Begin (Pick). Although only one branch will be actually executed, Begin(Pick) sends green tokens to all Pattern Templates in order to perform the dead-path-elimination on the branches that were not selected. End(Pick), similarly to End(Flow), waits for the green tokens from all branch patterns.

The While pattern

$$
\text { While } \rightarrow \text { Begin(While) PatternTemplate End(While) }
$$

differs from the Sequence pattern as Begin(While) has two green input lines and a guard condition. A green input token comes either from the pattern structurally preceding the while, or from End(While) in order to loop. Dually, End(While) outputs a green token either for the pattern structurally following the while, or for Begin(While). The guard condition is checked again by End(While) in order to avoid skipping the whole while in case of a false guard at the end of a cycle.

Although assign is a basic activity, it is translated with a structured pattern since it can contain several copy tags, each of which requiring a data exchange which may lead to a fault being raised. The Assign pattern:

$$
\text { Assign } \rightarrow \operatorname{Begin}(\text { Assign }) \text { Copy }^{+} \text {End(Assign) }
$$

has the same structure of the Sequence pattern, but it includes Copy patterns rather than arbitrary PatternTemplates. A Copy pattern is obtained from the basic pattern template by replacing the ActivitySpecificTask with a task named Copy, which inputs the "source" variable and which outputs the "target" variable. In this way, the assignment is achieved through the data mappings of the 


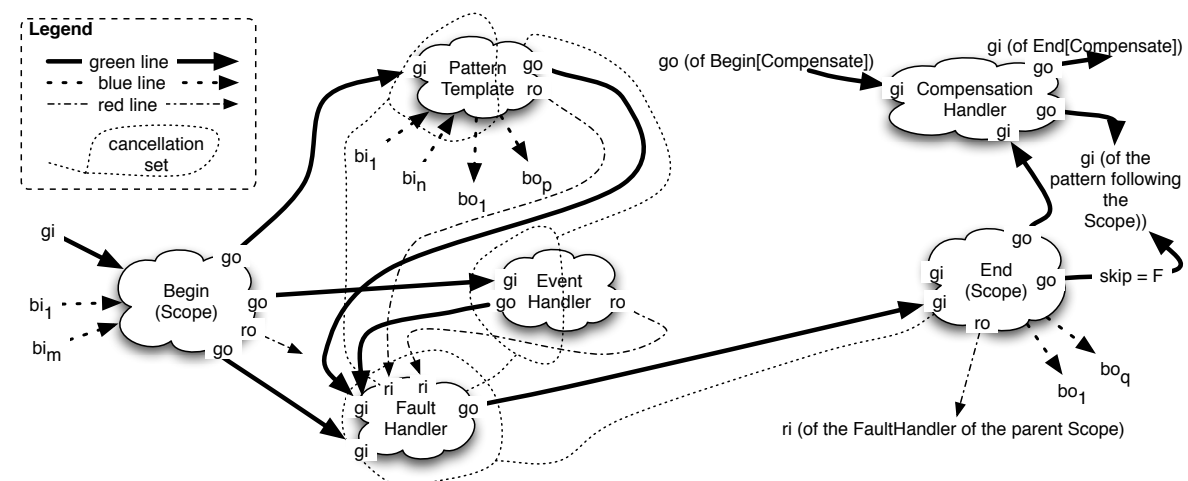

Fig. 2. High level view of the Scope pattern

Copy patterns. Furthermore, the Copy pattern does not have blue inputs and outputs.

A BPEL scope provides a specific context for an activity. It contains a (possibly default) fault handler, a (possibly default) compensation handler, as well as an optional event handler. The fault handler consists of one or more catch clauses for grabbing faults raised inside the scope. A catch is a container of an activity, guarded by a fault name and an optional fault variable. A catchAll has an always true guard and no fault name or variable. The compensation handler provides a (compensating) activity that can be invoked either explicitly (through a compensate), or implicitly (in case of a fault). The compensation handler is activated only when the scope finished its execution successfully. (In this paper we deal with explicit compensation only, due to the troublesome default compensation mechanism, e.g., compensating a scope inside a while). Last but not least, an event handler defines message and alarm events that can be triggered repeatedly and concurrently during the lifetime of the scope. The Scope pattern has the structure:

\section{Scope $\rightarrow$ Begin(Scope) PatternTemplate FaultHandler [CompensationHandler] [EventHandler] End(Scope)}

and the structural dependencies among the various patterns involved are illustrated in Figure 2. Begin(Scope) sends green tokens to the Pattern Template, to the EventHandler, and to the FaultHandler. The FaultHandler will further receive either one green token from the Pattern Template and one green token from the EventHandler (if any), or one red token from the PatternTemplate or from the EventHandler. In the former case, the entire FaultHandler will be skipped either because the Pattern Template was completed successfully, or because the entire scope has to be skipped. The latter case corresponds to a fault being raised (and uncaught) inside the Pattern Template, or inside the EventHandler. In case that the fault cannot be processed, the FaultHandler will send a green token to End(Scope), which has to send a red token further to the FaultHandler of the 
parent scope pattern (if any). Note that only the FaultHandler will forward a (green) token to End(Scope). End(Scope) is in charge of enabling the CompensationHandler if the Pattern Template was successful. It is important to note that End(Scope) has to save a copy of all the scope variables as required by the CompensationHandler. If the scope is skipped, End(Scope) has to clear the green tokens received by the FaultHandler from the PatternTemplate and from the EventHandler as they are redundant. Furthermore, in this case it is unnecessary to perform the dead-path-elimination inside the EventHandler as links cannot cross its boundary. However, we do have to perform the dead-path-elimination inside the FaultHandler.

The FaultHandler pattern has a similar structure to the Sequence pattern:

$$
\text { Begin(FaultHandler) PatternTemplate* End(FaultHandler) }
$$

except that each Pattern Template corresponds here to a catch activity and hence it has a guard condition checking the fault name and data. Furthermore, $B e$ gin(FaultHandler) uses a RedGate (instead of a BlueGate) that waits for red tokens to be sent (viz., faults to be raised) from inside the Pattern Template (or from inside the EventHandler) of its scope. In order to interrupt the normal execution of the scope in case of a fault being raised, the RedGate uses a cancellation set that includes all patterns of the scope's PatternTemplate and EventHandler except CompensationHandler patterns corresponding to scopes nested in its scope. If the BPEL process does not define a fault handler, the translator generates a default FaultHandler pattern consisting of Begin(FaultHandler) and End(FaultHandler) only. In this way, the faults received by this default FaultHandler will be forwarded (through EndScope) to the FaultHandler of the parent scope (or of the entire process). In the pattern of the EventHandler:

$$
\text { Begin(EventHandler) PatternTemplate }{ }^{+} \text {End(EventHandler) }
$$

the PatternTemplates execute concurrently, and each one is placed in a loop with a guard that checks the end of the Pattern Template pattern translating the activity inside the scope. Note that the scope's PatternTemplate is in charge of clearing all tokens of the Pattern Templates that implement alarms upon its completion. Finally, the CompensationHandler pattern is:

Begin(CompensationHandler) PatternTemplate* End(CompensationHandler) If the scope completes successfully, the Begin(CompensationHandler) is activated and waits for a green token from a Compensate pattern. Upon completion, the End(CompensationHandler) returns the green token to the Compensate. If a BPEL scope does not define a compensation handler yet there is a compensate activity targeting the respective scope, the translator generates a default CompensationHandler consisting only of Begin(CompensationHandler) directly linked to End(CompensationHandler).

Finally, the BPEL compensate is translated with the pattern:

\section{Begin(Compensate) End(Compensate)}

since compensate terminates only when the invoked CompensationHandler finishes its execution. Recall that we consider only simple explicit compensation, that is compensate activities specifying the name of the scope to be compensated, 
without considering scopes nested inside while activities. Begin(Compensate) sends a green token directly to End(Compensate) if the compensate is skipped, or if the scope to be compensated did not finish its execution. Otherwise, the green token is sent to the Begin(CompensationHandler) of the scope to be compensated. Dually, End(Compensate) receives a green token either directly from Begin(Compensate), or from the End(CompensationHandler) of the scope to be compensated. Then, it forwards it to the pattern structurally following the compensate. Further details on the Scope pattern will be commented in the Section dedicated to discussing an use case.

\subsection{BPEL Processes}

A BPEL process encapsulates the process activity and it can further define a fault handler, a compensation handler, as well as an event handler.

The Process pattern:

Begin(Process) FaultHandler [EventHandler] PatternTemplate End(Process) resembles the $S$ cope pattern, altohough there are several differences between the two. For example, Begin(Process) and End(Process) have to be connected to the input condition and to the output condition, respectively, of the workflow.

Begin(Process) enables the Pattern Template, the FaultHandler, as well as the EventHandler (if any). If the BPEL process does not define a FaultHandler, or if it does but it does not contain a catchAll clause, one (default) FaultHandler with a default catchAll (viz., an Empty pattern) must be defined in the Process pattern. This is needed to catch all uncaught faults being raised within the process. Note that the reception of a fault by the process FaultHandler leads to an abnormal process termination, even if the fault is processed. Furthermore, faults being raised (and uncaught) inside the process FaultHandler lead to the immediate execution of the End(Process) pattern, as in the case of a Terminate (see next). Differently from the Scope, there are no green tokens being sent from the PatternTemplate and from the EventHandler to the FaultHandler. This is due to the fact that the FaultHandler cannot be skipped because neither the Process can be skipped nor the dead-path-elimination must be employed inside its FaultHandler pattern. The Pattern Template and EventHandler forward each one green token to End(Process).

The EventHandler is active for the entire process lifetime and the PatternTemplate of the process is in charge of clearing its tokens upon its completion, similarly to a Scope. In order to minimise the number of cancellation sets defined in the workflow, all Terminate patterns forward the green token to End(Process), which is in charge of immediately terminating the entire business process. It does so by clearing all the tokens of the Pattern Template corresponding to the activity defined by the process. Hence, End(Process) is enabled if it receives either one green token from the process PatternTemplate and another from the EventHandler (if any), or one green token from a Terminate, or from the process FaultHandler.

The compensation handler can only be invoked by platform-specific means. Consequently, we do not consider a compensation handler for the entire busi- 
ness process. Furthermore, the process compensation handler would block the workflow waiting for a green token.

A BPEL process is translated into a YAWL workflow by instantiating the Process pattern. This leads to recursively instantiating the Begin(Process), FaultHandler, EventHandler (if any), and End(Process) patterns, as well as the Pattern Template corresponding to the process activity. Note that the instantiation of a pattern takes into account the context in which the activity is placed inside the BPEL process. Namely, instantiating a pattern means adjusting the (number of) input and output lines, setting and mapping the inputs and outputs of the tasks in the pattern, as well as suitably interconnecting its child patterns. The instantiating process bottoms-out at basic pattern templates.

\section{A Use Case}

Consider a simple BPEL process that computes the greatest common divisor (GCD) of two numbers. Basically, the GCD is computed by repeatedly raising an exception if one of the two numbers is bigger than the other and by decreasing its value in the corresponding catch. Due to space limitations we present hereafter a simplification of the BPEL process. Figure 3 gives the highlevel view of the YAWL workflow obtained from the GCD process. The interested reader is kindly asked to download the archive containing the full BPEL process as well as the YAWL workflow of the example from the following address: http://www.di.unipi.it/ ppopescu/GCDExample.zip.

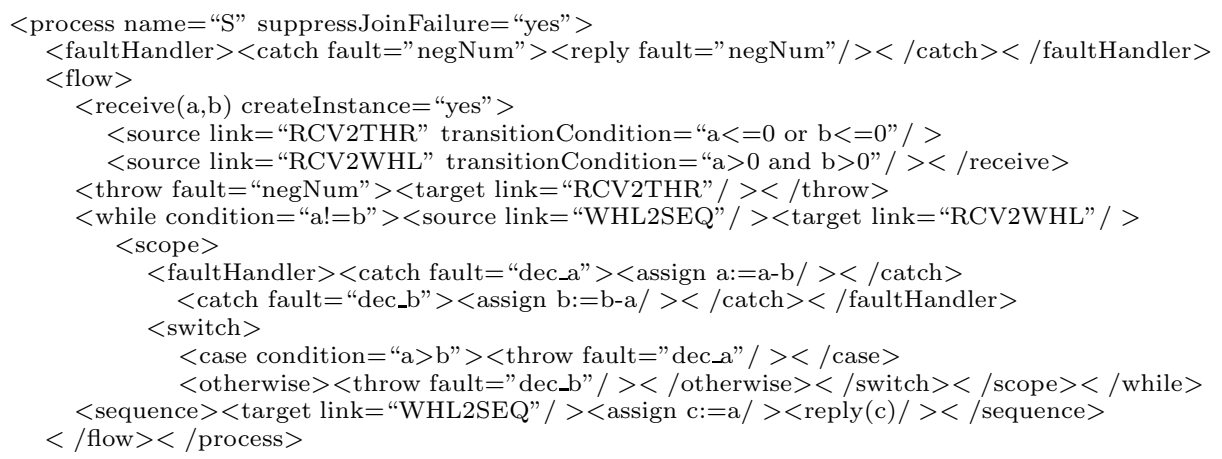

Consider an execution scenario in which the two input variables $a$ and $b$ take the values of 2 and 4, respectively. The workflow executes first Begin(Process) (that outputs two green tokens) followed by Begin(Flow) (that outputs four green tokens) and by Receive (that outputs one green token). As both numbers are strictly positive, Receive sends a blue token to Begin(While) and another blue (skipping) token to Throw. Because the suppressJoinFailure (set for the entire process only) has a yes value, skipping the Throw does not raise a joinFailure, but forwards the green token to End(Flow). The execution continues with $B e$ gin (While) and then with Begin(Scope) (as $a !=b)$ that forwards a green token to Begin(Switch) and another to the Begin(FaultHandler) of the scope. The first 


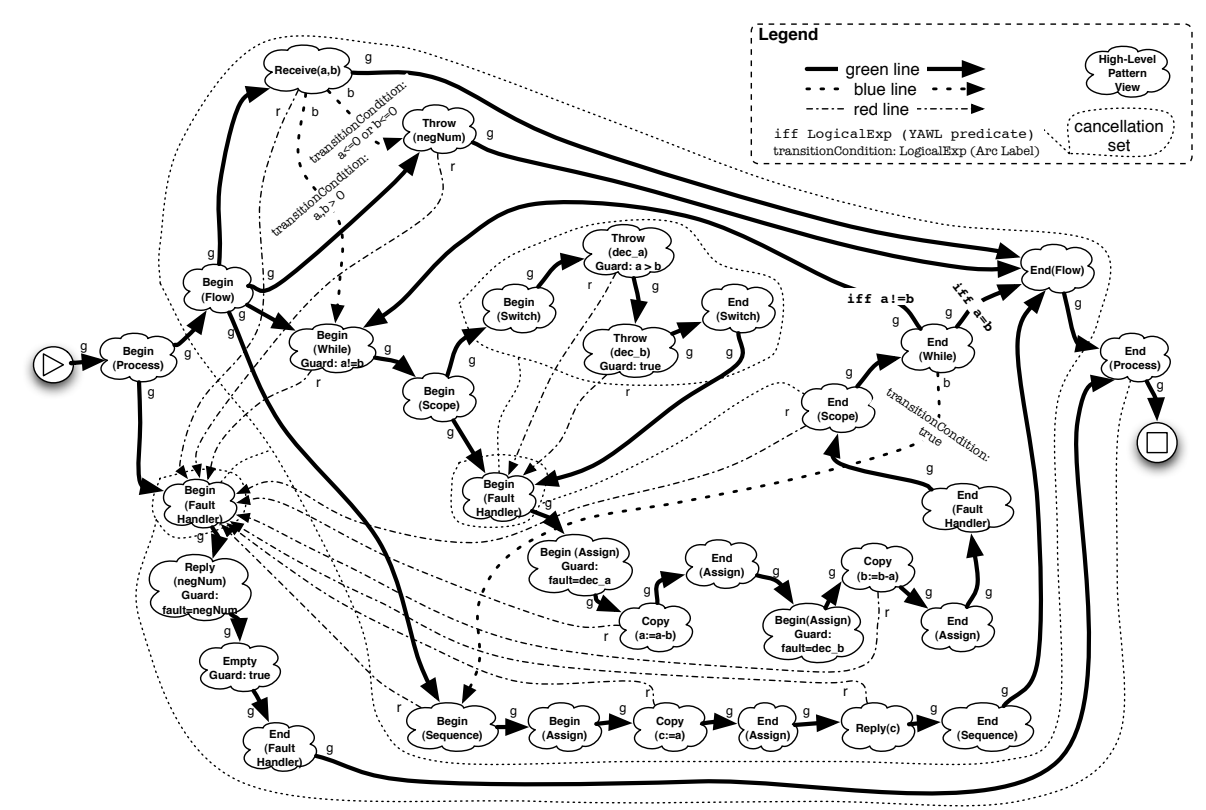

Fig. 3. YAWL workflow obtained from the GCD process

Throw in the switch is skipped as $a<b$, yet the second one (of the otherwise branch) is executed, and a $d e c_{-} b$ fault is raised. As a result, only a red token is sent further to the Begin(FaultHandler) of the scope (that clears all tokens of the Switch). The first Assign is skipped (as fault= "dec_b"), while the second Assign decreases the value of $b$ by $a$. The green token will reach next End(FaultHandler) and then $\operatorname{End}($ Scope) that forwards the green token to End(While) (as the fault was processed). Because $a=b=2, E n d($ While) sends a green token to End(Flow) and a blue token to Begin(Sequence). The execution of the Assign inside the Sequence leads to copying the value of $a$ into $c$ and to replying with the latter to the client. Finally, End(Sequence) outputs a green token that enables End(Flow), which has now gathered all its input (green) tokens. End(Flow) forwards a green token to End(Process) that first clears all input tokens of the Begin(FaultHandler) of the process, and then it sends the green token to the output condition, marking in this way the end of the workflow.

\section{Concluding Remarks}

We have outlined a methodology for translating BPEL processes into YAWL workflows. Its main strengths are that (1) it defines YAWL patterns for all BPEL activities, (2) it provides a compositional approach to construct structured patterns from suitably interconnecting other patterns, and (3) it handles events, faults and (explicit) compensation. 
Fisteus et al. [1] describe VERBUS, a FSM-based framework for the formal verification of BPEL processes, but they do not treat synchronisation links, complex fault handling, as well as event and compensation handling. Koshkina and van Breugel [6] introduce the BPE-calculus in order to formalise the control-flow of BPEL and build upon it a tool for the analysis (e.g., deadlock freedom) of business processes. Still, they do not tackle fault and compensation handling. Hinz et al. [5] give a PN semantics to BPEL processes by defining a pattern for each BPEL activity. However, they abstract from data and leave out transition guards. Consequently, control-flow decisions based on the evaluation of data are replaced by non-deterministic choices. Our approach does not suffer from this limitation as both BPEL and YAWL use XMLSchema and XPath for data manipulation, and hence the data translation between the two is straightforward. Ouyang et al. 7] formalise BPEL in terms of PNs with the purpose of analysing its control-flow. Although they handle both synchronisation links and exceptional behaviour, their focus is on the analysis, and not on the composition of business processes.

We believe that the translation described in this paper constitutes an important brick for the development of formal analysis and transformations of BPEL processes. It also directly contributes to our long-term goal of aggregating and adapting heterogeneous Web services [43]. In this perspective, our next step will be the integration of our Java prototype implementation of the BPEL2YAWL translator with the Java implementation of the core aggregation mechanism of [4], in order to yield a single tool supporting the disciplined, semi-automated aggregation of BPEL services. A further line for future work is the development of other translators to convert other types of Web service descriptions (e.g., OWL-S) into YAWL.

\section{References}

1. J. Arias-Fisteus, L. S. Fernández, and C. D. Kloos. Formal Verification of BPEL4WS Business Collaborations. In K. Bauknecht, M. Bichler, and B. Pröll, editors, EC-Web, volume 3182 of $L N C S$, pages 76-85. Springer, 2004.

2. BPEL4WS Coalition. Business Process Execution Language for Web Services (BPEL4WS) Version 1.1. (ftp://www6.software.ibm.com/software/developer/ library/ws-bpel.pdf).

3. A. Brogi and R. Popescu. Service Adaptation through Trace Inspection. In S. Gagnon, H. Ludwig, M. Pistore, and W. Sadiq, editors, Proceedings of SOBPI'05, pages 44-58, 2005. (http://elab.njit.edu/sobpi/sobpi05-proceedings.pdf).

4. A. Brogi and R. Popescu. Towards Semi-automated Workflow-Based Aggregation of Web Services. In B. Benatallah, F. Casati, and P. Traverso, editors, ICSOC'05, volume 3826 of $L N C S$, pages 214-227. Springer, 2005.

5. S. Hinz, K. Schmidt, and C. Stahl. Transforming BPEL to Petri Nets. In W. van der Aalst, B. Benatallah, F. Casati, and F. Curbera, editors, Proceedings of the Third International Conference on Business Process Management (BPM 2005), volume 3649 of LNCS, pages 220-235, Nancy, France, Sept. 2005. Springer-Verlag.

6. M. Koshkina and F. van Breugel. Verification of business processes for Web services. Technical Report CS-2003-11, York University, October 2003. (http:// www.cs.yorku.ca/techreports/2003/CS-2003-11.ps). 
7. C. Ouyang, E. Verbeek, W. M. van der Aalst, S. Breutel, M. Dumas, and A. H. ter Hofstede. Formal Semantics and Analysis of Control Flow in WS-BPEL. Technical Report 2174, Queensland University of Technology, February 2006. Available from: http://eprints.qut.edu.au/archive/00002174/01/BPM-05-15.pdf.

8. OWL-S Coalition. OWL-S: Semantic Markup for Web Services Version 1.1. (http://www.daml.org/services/owl-s/1.1/overview/).

9. M. P. Papazoglou and D. Georgakopoulos. Service-Oriented Computing. Communication of the ACM, 46(10):24-28, 2003.

10. W. M. P. van der Aalst and A. H. M. ter Hofstede. YAWL: Yet Another Workflow Language. Inf. Syst., 30(4):245-275, 2005.

11. W. M. P. van der Aalst, A. H. M. ter Hofstede, B. Kiepuszewski, and A. P. Barros. Workflow Patterns. Distrib. Parallel Databases, 14(1):5-51, 2003.

12. WSDL Coalition. Web Service Description Language (WSDL) version 1.1. (http://www.w3.org/TR/wsdl). 